\title{
I-Xe ages of chondrites and their relationship to solar-wind derived noble gas concentrations
}

\author{
KOHARU ARAI ${ }^{1}$, ATSUSHI TAKENOUCHI ${ }^{2}$, HIROCHIKA \\ SUMINO $^{3}$ AND SHOGO TACHIBANA ${ }^{4}$ \\ ${ }^{1}$ Univ. Tokyo \\ ${ }^{2}$ National Institute of Polar Research \\ ${ }^{3}$ The University of Tokyo \\ ${ }^{4}$ Dept. Earth Planet. Sci., Univ. Tokyo \\ Presenting Author: arai-koharu@g.ecc.u-tokyo.ac.jp
}

The lifetime of protoplanetary disk gas ranges 1-10 million years, which depends on viscous dissipation and photoevaporation of the disk gas. It is not clear how long the Sun's protoplanetary disk held its gas component, during which gas giants formed. Bajo [1] found a correlation between Solar-wind derived noble gas concentrations and I-Xe ages of brecciated chondrites. Bajo [1] hypothesized that the I-Xe ages, which could represent impact ages [2], of solar-wind-rich meteorites indicated the timing of disk-gas clearing because the solar wind reaches to the surface of small bodies only after the complete dissipation of disk gas. In this study, to test this hypothesis, I-Xe ages and solar-wind noble gas concentrations of Zag (H36), Northwest Africa 801 (CR2) (NWA 801), Ochansk (H4), Nuevo Mercurio (H5) meteorites were determined.

About $30 \mathrm{mg}$ fragments of these meteorites, irradiated with neutrons at the Kyoto University research reactor, were heated in vacuum stepwisely in the temperature range of $400-1800^{\circ} \mathrm{C}$ to extract xenon. After purifying the noble gases, xenon isotope ratios were measured using a magnetic-sector-type mass spectrometer VG3600. The obtained ${ }^{129} \mathrm{Xe} /{ }^{128} \mathrm{Xe}$ ratios of the samples were converted to relative I-Xe ages by comparing with ${ }^{129} \mathrm{Xe} /{ }^{128} \mathrm{Xe}$ ratio of the Shallowater meteorite with the absolute age of $4.5633 \pm 0.0004$ billion years [3]. About $5 \mathrm{mg}$ fragments of these meteorites were heated in vacuum at 800 and $1700^{\circ} \mathrm{C}$ for noble gas analysis as well. We found that the solar-wind-poor portion of Zag was systematically older (4.551 \pm 0.008 and $4.558 \pm 0.013 \mathrm{Ga})$ than the solar-wind-rich portion $(4.541 \pm 0.010$ and $4.551 \pm 0.006 \mathrm{Ga}$ ) as shown in [1]. The solar wind-rich meteorite NWA 801 shows the I-Xe age of $4.529 \pm 0.016 \mathrm{Ga}$. The present dataset is broadly consistent with the hypothesis [1], but further data with lesser analytical uncertainties is surely required.

References: [1] K. Bajo (2010) Ph.D thesis, Univ. Tokyo. [2] J. D. Gilmour and M. J. Filtness (2019) Nature Astronomy 3, 326. [3] J. D. Gilmour et al. (2006) Meteorit. Planet. Sci. 41, 19. 